\title{
Fair power and transmission rate control in wireless networks
}

\author{
Eitan Altman \\ Maestro project, INRIA \\ Sophia-Antipolis, France \\ Email: eitan.altman@inria.fr
}

\author{
Jérôme Galtier \\ France Telecom R\&D \\ Sophia-Antipolis, France \\ Email: jerome.galtier@francetelecom.fr
}

\author{
Corinne Touati \\ Computer Science Department \\ University of Tsukuba, Japan \\ Email: corinne@osdp.cs.tsukuba.ac.jp
}

\begin{abstract}
In third generation mobile networks, transmission rates can be assigned to both real time and non real time applications. We address in this paper the question of how to allocate transmission rates in a manner that is both optimal and fair. As optimality criterion we use the Pareto optimality notion, and as fairness criterion we use a general concept of which the max-min fairness (which is the standardized fairness concept in ATM networks) and the proportional fairness (which characterizes fairness obtained by some transport protocols for the Internet) are special cases. We show that the problem is a joint optimization system of the transmission rate and the power. We formulate the fair allocation problem as an optimization problem and propose both exact as well as approximating solutions. We consider both uplink and downlink problems and study also macrodiversity.
\end{abstract}

\section{INTRODUCTION}

Rate control of calls is an important network management issue in third generation mobile networks. Indeed, not only data transfer but also real time audio and video applications can be transmitted at various rates by selecting an appropriate Codec. In the case of voice applications, UMTS will use the Adaptive Multi-Rate (AMR) codec that offers eight different transmission rates of voice that vary between $4.75 \mathrm{kbps}$ to 12.2 $\mathrm{kbps}$, and that can be dynamically changed every $20 \mathrm{msec}$. Of course the transmission rate has an impact on the perceived quality. The reduction of the transmission rate is necessary for maintaining a call whose received energy per bit is too small, and it allows to maintain a larger number of calls in the system; it will be studied here in conjunction with power control which is yet another tool that can be used to increase the received energy per bit (but which also has an impact on the interference experienced by other calls).

A well studied problem is that of choosing transmission rates so as to maximize the system's throughput, see [1]. Alternatively, if two mobiles $A$ and $B$ transmitting at the same rate have the same received power at their base station and if $A$ has larger attenuation than $B$, then it transmits with larger power than $B$, thus causing more interference than $B$ in the base stations of neighboring cells. Hence systemwise, it is profitable to assign to mobile $A$ lower throughput than to mobile $B$ if there are not sufficient radio resources to assign the maximum throughput to both. This suggests large differences in throughputs assigned to mobiles according to their attenuation level when seeking the system optimal viewpoint.
Yet, a second important consideration in assigning throughputs in networks is fairness. Several fairness concepts have been suggested and implemented in various network architectures, but let us first recall the concept of global optimization. Global optimization Consider a system with $N$ transmitting source. Let $\underline{r}=(r(1), \ldots, r(N))$ be a assignment vector of transmission rates. The global optimization maximizes the total throughput $\sum_{n \in N} r(n)$. It can lead to situations in which the allocation is null for one or several sources, and is therefore not consider as fair.

Max-min fairness In ATM networks, the standardized fairness concept in traffic whose rate is controlled (the ABR - Available Bit Rate class) is the so called "max-min fairness" [2]. An assignment vector of rates is said to be max-min fair if one cannot increase the assignment of a source $i$ without decreasing the assignment of a source $j$ for which $r(j) \leq r(i)$ [2], [3]. The quantity that is assigned fairly in ATM is the excess of the throughput beyond a prenegociated minimum transmission rate. Another related standardized fairness concept in ATM is the "weighted max-min fairness" in which the quantity that is to be assigned fairly is the excess throughputs (beyond the minimum guaranteed) weighted by some multiplicative constant depending on the connections.

Proportional fairness In the Internet, the large majority of transfers use the TCP/IP transport control mechanism. The assignment of throughput to various connections using TCP/IP (and other related protocols) can be described using the concept of "proportional fairness" as was shown in [4]. An assignment $r$ is said to be proportionally fair if it is feasible (satisfies the system's constraints) and if for any other feasible assignment $\underline{r}^{*}$, the aggregate of proportional changes is nonpositive:

$$
\sum_{i=1}^{N} \frac{r^{*}(i)-r(i)}{r(i)} \leq 0 .
$$

The proportional fairness is known to maximize the quantity $\prod_{i} r(i)$. Equivalently, it is an assignment that maximizes $\sum_{i} \log r(i)$. A (weighted version of the) proportional fairness is also advocated for future developments of TCP, see e.g. [5]. The way TCP shares bandwidth between connection has become a reference for other real-time applications over the Internet that do not use TCP; such applications are called "TCP friendly". 
Both proportional fairness and max-min fairness possess optimality properties: they are both Pareto optimal ${ }^{1}$. The proportional fairness is a good compromise between the system global optimum (i.e. the sum of all mobiles' rates) and the welfare maximization approach of max-min fairness.

Generalized fairness criterion It has been shown in [6] that all three approaches: the system optimization, the max-min fairness and the proportional fairness are all special cases of a generalized fairness concept. Given a positive constant $\alpha \neq 1$, consider the optimization problem:

$$
\operatorname{Maximize} \sum_{i} \frac{r(i)^{1-\alpha}}{1-\alpha}
$$

subject to the problem's constraints. Assume that the $r(i)$ are defined on a convex set. Then since the objective function is concave and the constraints are linear, this defines a unique allocation which is called the $\alpha$-fair allocation. It turns out [6] that this allocation corresponds to the globally optimal allocation when $\alpha \rightarrow 0$, to the proportional fairness when $\alpha \rightarrow 1$, to the harmonic mean fairness (another well known fairness concept) when $\alpha \rightarrow 2$, and to the max-min allocation when $\alpha \rightarrow \infty$.

We should mention that other aspects of fair resource assignment in wireless networks have been studied previously. These were aspects related to scheduling back logged packets [7]-[9], so as to achieve already given average transmission rates of different sources. Our study aims, in contrast, to fairly assign the transmission rates.

\section{Our main contributions are}

1. A methodology for combined rate and power control for achieving arbitrary tradeoff between fairness and system's global maximum for both downlink and uplink as well as for macrodiversity.

2. Transforming a non convex optimization problem into a convex problem with linear constraints. This extends our results in [10] in which only the uplink was considered and which proposed only an approximation in order to derive a convex optimization problem.

The structure of the paper is as follows. We first mention in the next section some other works on rate control in wireless networks (not directly related to fairness concepts). We then introduce in Section III our model and show how the fair assignment problem can be formulated as one of two possible optimization problems: one in which transmission rate can be assigned any real value within some given interval, and one in which finitely many transmission rates are available for each mobile. We analyze the properties of the system in Section IV. We then apply our results to the uplink problem in Section V and propose exact and approximating solutions. The downlink problem is examined in Section VI and the macrodiversity is studied in Section VII. Numerical results are given in Section VIII. A concluding section ends this paper with extensions to utility-based fairness concepts.

\footnotetext{
${ }^{1}$ An assignment $\underline{r}$ is Pareto optimal if one cannot increase the assignment of one source $i$ without strictly decreasing an assignment of another source $j$
}

\section{RELATED WORK ON RATE CONTROL IN WIRELESS} NETWORKS

We briefly mention in this section some recent papers on rate control in CDMA wireless systems.

The paper that mostly relates to ours is [1]. It considers the problem of optimizing transmission rates and powers. Discrete available rates. The problem is formulated mathematically as a mixed linear-integer programming, for which polynomial optimization algorithms are not available. A heuristic approximation approach based on a Lagrangian is proposed and tested.

Another related paper is [11], where the authors study the optimal control of both the power, and spreading gain (the latter is equivalent to controlling the throughput). The authors restrict to a single cell and to the uplink. The model includes channel coding (FEC, Forward Error Correction), and a general function for BER (Bit Error Rate) as a function of the SIR (Signal to Interference Ratio). Similar models are studied in [12], [13].

Several papers study optimizing throughput assignment for non-real time traffic.

In [14], the quantity that is maximized is the effective data rate: the transmission rate times the BER, where the latter is a function of the SIR and the transmission rate. A Lagrangian approach is used. There is no a priori target SIR. This approach is useful for NRT (Non Real Time) traffic. Note that if we assign to a source a transmission of rate $R$ with 0 percent losses, the effective throughput is the same as if we transmitted at $2 R$ and lost half the information. In contrast to NRT, for RT (Real Time) applications, the two scenarios would give different utility (quality perception). In [14] the function of the SIR is taken in an example to be the Shannon capacity; note that approaching that capacity requires long blocks of codes which makes the scheme not useful for RT. There is also a part that considers both RT and NRT (sec VI) but only the throughput of NRT is optimized.

In [15], the authors consider uplink CDMA with two classes, mobiles of the first class (RT) transmit all the time, the other mobiles (NRT) are time-shared. The benefits of time sharing is studied as well as the conditions for silencing some, where one of the studied objectives is that of maximizing throughputs (while keeping the SIR at acceptable levels). The paper takes into account that when a mobile is silent, it still requires energy (for synchronization). We also note that the amount of information to transmit is not changed by the scheduling.

In [16], the author studies the Erlang capacity as a function of the throughputs assigned to NRT applications. Unlike the framework in [1], which we adopt in this paper as well for RT applications, the volume of information transmitted by NRT applications (such as a file transfer) is not affected by the assigned throughput. Thus a static optimization problem, as done in previously mentioned papers, is not adequate to describe the effect of throughput assignment for NRT applications. The model in [16] takes into account the impact of throughput assignment on the call duration in order to 
compute the Erlang capacity as a function of the assigned throughput.

Another related research direction has been the assignment of instantaneous rate of packet transmission at the buffers in CDMA wireless systems. In these papers, the actual transmission rate of the source is not controlled.

In [17] the packet transmission rates at the link layer buffers is allocated at as a function of the traffic profiles and is computed according to required bounds on packet losses at these buffers. The paper uses effective bandwidth notions for CBR/VBR traffic (Constant/Variable Bit Rate), and others for ABR (Available Bit Rate). Some other closely related papers are [18]-[20].

In [21], the author considers combined power and rate control of each of a number of queue so as to minimize power and delay. In the considered model, the power and rate assignments determine loss probabilities and there is a given constraint on the loss rate. No retransmissions and no scheduling are considered.

In [22], the authors describe the feasible set of powers/rates in a single cell. They show that this is not a convex set. Convexification is possible by an appropriate time sharing or scheduling of packets. The results are used for assigning transmission rates at the buffers (again, the source rates are given and not controlled), so as to achieve required bounds on delays. The scheduling decisions are taken according to the traffic profile of each mobile which is characterized by the average rate and the burstiness (the so-called $\sigma-\rho$ constraints).

Among all the research directions we mentioned above, our paper is related to the first two references as it is concerned with the actual assignment of transmission throughput rates at the sources (rather than inside the network) of real time applications. We consider a multicell environment with particular attention on uplink and downlink rate control, and include a study on macrodiversity. (This is in contrast to [11] who considers only the uplink control in a single link, or [1] whose framework seems more adapted to the uplink multicell case.) Yet, an important feature of our paper is the introduction of new fairness considerations into the rate allocation problem.

\section{THE MODEL}

We use the notations of [1] applicable for both the uplink and the downlink and extend their model. Consider a cellular radio system with $S$ transmitting sources. Source $s$ can transmit with total power $p_{s, t o t}$ within the interval $\left[0, \overline{p_{s, t o t}}\right]$. In the following, if source $s$ has different channels, $p_{s, t}$ will denote the power of the signal emitted by source $s$ to destination $t$. Also, $p_{N C, s}$ represents the power of the non-power controlled channels. Therefore:

$$
p_{s, t o t}=\sum_{t} p_{s, t}+p_{N C, s} .
$$

We take the following notations: $m \quad$ a mobile unit

$b \quad$ a base station $m, b)= \begin{cases}(s, t) & \text { in the uplink case } \\ (t, s) & \text { in the downlink case }\end{cases}$

$c_{m} \quad$ the cell of mobile $m$

$M \quad$ the number of mobile units

$N_{c} \quad$ the number of mobiles in cell $c$

$N(m, b)$ the background noise power at the receiver (it represents thermal noise and also radio interference from non-power controlled channels.)

$C \quad$ a multiplicative constant

$p_{m, b}^{\prime} \quad$ the normalized power: $\exists K_{s, t}, p_{m, b}^{\prime}=K_{s, t} p_{s, t}$. We will show that :

$K_{s, t}= \begin{cases}g_{m, b} & \text { (the link gain) in the uplink, } \\ 1 & \text { in the downlink. }\end{cases}$

Given a power vector $P=\left(p_{1, t o t}, \ldots, p_{s, t o t}\right)$, the received signal to interference ratio of mobile $m$ is given by

$$
\operatorname{SIR}_{m}(P)=\frac{p_{m, b}^{\prime}}{N(m, b)+C \sum_{\substack{m^{\prime} \neq m \\ m^{\prime} \in c_{m}}} p_{m^{\prime}, b}^{\prime}} \quad 1 \leq m \leq M .
$$

As $p_{s, t o t}^{\prime}$ is bounded, then $p_{m, b}^{\prime}$ is bounded by a value that we will denote by $\overline{p_{m, b}^{\prime}}$. The values of $C, N$ and $p^{\prime}$ will be expressed in Section V for the uplink and Section VI for the downlink.

As explained in [1], the above model can be useful for both uplink and downlink. However, we shall later use the particular structure of the uplink and of the downlink in order to simplify the solution.

We next describe two possible settings for the power and transmission rate control.

\section{A. The continuous model}

In the first model, mobile $m$ can use any value of throughput between a minimum guaranteed value $M R_{m}$ and a maximum value $P R_{m}$. This can be achieved if a packet mode is used with an appropriate scheduling (see e.g. [23], [24] and references therein). Denote $r(m)$ the transmission rate assigned to mobile $m$ and $R=(r(1), \ldots, r(M))$ the rate vector. We assume that, for each mobile, there is a minimum required value of $S I R_{m}$ per transmitted bit per second, which we denote by $\delta_{m}$.

Let $\left(E_{b} / I_{o}\right)_{i}$ be the ratio of bit energy to interference power spectral density of mobile $m$, and $W_{m}$ the spreading bandwidth at chip rate for mobile $m$. We then have

$$
\delta_{m} \leq \frac{1}{W_{m}}\left(\frac{E_{b}}{I_{o}}\right)_{m}=\frac{\operatorname{SIR}_{m}(P)}{r(m)},
$$

implying $\delta_{m} r(m) \leq \operatorname{SIR}_{m}(P) .^{2}$

\footnotetext{
${ }^{2}$ Note that we implicitly assume that $\left(E_{b} / I_{o}\right)_{m}$ does not depend on the transmission rate $r(m)$. This is a standard assumption in modeling literature, see e.g. [1]. In practice, however, it may depend on $r(m)$, see e.g. [25, p. $151,222,239]$. But as we see from [25, Fig. 10.4, p. 222], it is close to a constant throughout long range of bit rates. For example, between $16 \mathrm{Kbps}$ and $256 \mathrm{Kbps}$, the maximum variation around the median value is less than $20 \%$. We thus propose to take for the value of $\left(E_{b} / I_{o}\right)_{m}$ its average or median value over the range $\left[M R_{m}, P R_{m}\right]$. However, if the exact dependence is available analytically, it can be included into our model.
} 
Thus the solution of our joint power and transmission rate assignment problem is constrained to belong to the set $\Pi^{c}=$ $(P, R)$, defined through:

$$
\left\{\begin{array}{cl}
0 \leq p_{s, t o t} \leq \overline{p_{s, t o t}}, & 1 \leq s \leq S, \\
M R_{m} \leq r(m) \leq P R_{m}, & 1 \leq m \leq M . \\
\delta_{m} r(m) \leq \frac{p_{m, b}^{\prime}}{N(m, b)+C \sum_{m^{\prime} \neq m} p_{m^{\prime}, b}^{\prime}}, &
\end{array}\right.
$$

A fair allocation can now be obtained using the following optimization problem:

$$
\text { Find }(P, R) \in \Pi^{c} \text { that Maximizes } \sum_{m=1}^{M} \frac{r(m)^{1-\alpha}}{1-\alpha} \text {. }
$$

\section{B. The discrete model}

There is a finite number of available transmission rates for each mobile. Let $r_{m}^{1}<r_{m}^{2}<\ldots<r_{m}^{K(m)}$ be the available transmission rates for mobile $m$.

One way to formulate the discrete model is to use the continuous model and add a constraint on the discrete values that the throughputs can have:

$$
\begin{aligned}
& \text { Find }(P, R) \in \Pi^{c} \text { that Maximizes } \sum_{m=1}^{M} \frac{r(m)^{1-\alpha}}{1-\alpha} \\
& \text { with } r(m) \in\left\{r_{m}^{1}, r_{m}^{2}, \ldots, r_{m}^{K(m)}\right\}
\end{aligned}
$$

We present below an alternative formulation of the problem following [1].

To properly receive messages at transmission rate $r_{m}^{k}$ with tolerable error probability, mobile $m$ is expected to attain an $\operatorname{SIR}_{m}(P)$ not less than a target $\gamma_{m}^{k}$.

Let $Y=\left(y_{m}^{k}\right)$ be a $0-1$ matrix such that for every mobile $m$ and rate $r_{i}^{k}$,

$$
y_{m}^{k}= \begin{cases}1, & \text { if mobile } m \text { is transmitting with rate } r_{m}^{k}, \\ 0, & \text { otherwise }\end{cases}
$$

Introduce arbitrarily chosen constants $A_{m}^{k}$ that represent the transmission power that mobile $m$ needs in order to attain $\gamma_{m}^{k}$, regardless of the interference power. More precisely, they can be chosen arbitrarily so as to satisfy

$$
A_{m}^{k} \geq \gamma_{m}^{k}\left(N(m, b)+C \times \sum_{\substack{m^{\prime} \neq m \\ m^{\prime} \in c_{m}}} \overline{p_{m^{\prime}, b}^{\prime}}\right)
$$

for all $m$ and $k$ (we allow in the definition to have $A_{m}^{k} \geq$ $\left.\overline{p_{m, b}^{\prime}}\right)$. Hence the constants $A_{m}^{k}$ satisfy

$$
A_{m}^{k} \geq \max _{P} \frac{p_{m, b}^{\prime} \gamma_{m}^{k}}{S I R_{m}(P)}
$$

which is in fact the condition that defines these constants in [1].
Then the solution of our joint power and transmission rate assignment problem is constrained to belong to the set $\Pi^{d}=$ $(P, Y)$ such that:

$$
\left\{\begin{array}{cc}
0 \leq p_{s, t o t} \leq \overline{p_{s, t o t}}, & s=1, \ldots, S, \\
y_{m}^{k} \in\{0,1\}, \quad \sum_{k=1}^{K_{i}} y_{m}^{k} \leq 1, & m=1, \ldots, M . \\
p_{m, b}^{\prime}+\left(1-y_{m}^{k}\right) A_{m}^{k} \geq \frac{p_{m, b}^{\prime} \gamma_{m}^{k}}{S I R_{m}(P)} . &
\end{array}\right.
$$

The first constraint represents the power constraints. The second states that at most one bit rate can be allocated to a mobile. The third constraint reduces to the constraint on $S I R_{m}$ when $r_{m}^{k}$ is the rate allocated to mobile $k$. For $r_{m}^{k}$ which is not the allocated rate, the inequality constraint is just a consequence of the definition of $A_{m}^{k}$.

A fair allocation can now be obtained using the following optimization problem:

Find $(P, Y) \in \Pi^{d}$ that Maximizes $\sum_{m=1}^{M} \frac{\left(\sum_{k=1}^{K} y_{m}^{k} r_{m}^{k}\right)^{1-\alpha}}{1-\alpha}$.

In this section we made explicit the system of equations corresponding to the continuous and the discrete models. In particular, we showed the need for a join allocation of rates $R$ and power $P$ vectors. In the following section, we focus on the first model.

\section{PROPERTIES OF THE SYSTEM}

\section{A. Equivalent problem}

We provide here an equivalent formulation of the problem based on a simple change of variables.

Lemma 1: The continuous problem (see section III-A) is equivalent to finding $(P, \mathcal{C})$ in $\hat{\Pi}^{c}=(P, \mathcal{C})$ that maximizes $Z(\mathrm{C}):=\sum_{m=1}^{M} \frac{1}{1-\alpha}\left(\frac{\rho(m)}{1-\delta_{m} \rho(m)}\right)^{1-\alpha}$ where $\hat{\Pi}^{c}=(P, \mathcal{C})$ is given by:

$$
\left\{\begin{array}{cl}
0 \leq p_{s, t o t} \leq \bar{p}_{s, \text { tot }}, & 1 \leq s \leq S, \\
\frac{M R_{m}}{1+\delta_{m} M R_{m}} \leq \rho(m) \leq \frac{P R_{m}}{1+\delta_{m} P R_{m}}, & 1 \leq m \leq M . \\
\delta_{m} \rho(m) \leq \frac{p_{m, b}^{\prime}}{N(m, b)+C \sum p_{m^{\prime}, b}^{\prime}}, &
\end{array}\right.
$$

Proof: Let $\mathcal{C}$ be the $N$-dimensional vector such that $\forall m=1 \ldots M, \rho(m)=\frac{r(m)}{1+C \delta_{m} r(m)}$. We should notice that $\delta_{m} \rho(m)=1-\frac{1}{1+\delta_{m} r(m)}$ and simply make the change of variables from $R$ to $\mathcal{C}$ in the inequalities (5).

Lemma 2: The objective function $Z$ is concave if for any $\mathcal{C}$ in the set of feasible solutions, we have: $\forall m, 1 \leq m \leq M$, $2 \delta_{m} \rho(m) \leq \alpha$. 
Proof: Note that the denominator $1-\delta_{m} \rho(m)$ is nonnegative over the feasible solutions (from the second inequality of system (5)). To determine whether the objective function is concave, we differentiate it twice with respect to $\rho(m), m=1, \ldots, N_{c}$ and obtain $\frac{\partial^{2} Z(\rho)}{\partial \rho(m)^{2}}=$ $\frac{2 \delta_{m} \rho(m)-\alpha}{\left(1-\delta_{m} \rho(m)\right)^{3} \rho(m)}\left(\frac{\rho(m)}{1-\delta_{m} \rho(m)}\right)^{-\alpha}$. This is nonpositive for all feasible $\rho(m)$ if $2 \delta_{m} \rho(m) \leq \alpha$.

Remark 1: A sufficient condition for the objective function to be concave is $\alpha \geq 2$. This condition can further be weakened. Let

$$
w=\max _{m=1, \ldots, N_{c}} \frac{\delta_{m} P R_{m}}{1+\delta_{m} P R_{m}} .
$$

Then a weaker sufficient condition for the objective function to be concave is that $\alpha \geq 2 w$. Quite often $w$ is close to zero (see e.g. discussion before Lemma 1 in [26]).

In the following, we will call acceptable transmission rate vector any vector $R$ (respectively $\mathcal{C}$ ) that accepts at least one feasible assignment $P$ satisfying the constraints (5)(respectively (9)).

\section{B. Properties of acceptable rate vectors}

We should start by noticing that:

Lemma 3: For any feasible $\mathcal{C}$ and for any cell:

$$
C \sum_{m^{\prime}} \delta_{m^{\prime}} \rho\left(m^{\prime}\right)<1
$$

Proof: Let $c$ be a cell and $b$ its associated base station. Consider the last inequality of system (9):

$$
\forall m, \delta_{m} \rho(m) \leq \frac{p_{m, b}^{\prime}}{N(m, b)+C \sum_{m^{\prime}} p_{m^{\prime}, b}^{\prime}} .
$$

As $N(m, b)>0$, then $\delta_{m} \rho(m)<\frac{p_{m, b}^{\prime}}{C \sum_{m^{\prime}} p_{m^{\prime}, b}^{\prime}}$. By summation, $C \sum_{m^{\prime}} \delta_{m^{\prime}} \rho\left(m^{\prime}\right)<\frac{C \sum_{m^{\prime}} p_{m^{\prime}, b}^{\prime}}{C \sum_{m^{\prime}} p_{m^{\prime}, b}^{\prime}}=1$.

Lemma 4: Consider the last inequality of system (9) when replaced by equality. We obtain: For a given cell $c$ with base station $b, \forall m \in c$,

$$
\delta_{m} \rho(m)=\frac{p_{m, b}^{\prime}}{N(m, b)+C \sum p_{m^{\prime}, b}^{\prime}},
$$

Then one can prove that this linear system of $N_{c}$ equations of $N_{c}$ variables admits one and only one solution $P P$ for any feasible $\mathcal{C}$.

Proof: It is sufficient to prove that the $N_{c}$ equations are linearly independent. They can be written as: $\forall m, \delta_{m} \rho_{m} N(m, b)=p_{m, b}-C \delta_{m} \rho_{m} \sum_{m^{\prime}} p_{m^{\prime}, b}$ It is of the form $A X=Y$ with: $Y=\left(\delta_{i} \rho_{i} N(i, b)\right)_{i}, \quad X=\left(p_{i, b}\right)_{i}$ and $A=I d_{N_{c}}-B$ with $I d_{N_{c}}$ the identity matrix of size $N_{c}$ and
$B$ the matrix

$$
B=\left(\begin{array}{ccc}
b_{1} & \ldots & b_{1} \\
b_{2} & \ldots & b_{2} \\
& \cdots & \\
b_{N_{c}} & \cdots & b_{N_{c}}
\end{array}\right) .
$$

with $\forall i, b_{i}=C \delta_{i} \rho_{i}$. If $U$ is an eigenvector of $A$ associated to eigenvalue $\lambda$, then $A U=\lambda U=U-B U$. Therefore $U$ is an eigenvector of $B$ with eigenvalue $1-\lambda$. But $\operatorname{rank}(B)=1$ and $\operatorname{trace}(B)=\sum_{i} b_{i}$. Then $A$ has only two eigenvalues that are 1 and $1-C \sum_{i} \delta_{i} \rho_{i}$. Therefore $A$ is singular $\left(1-C \sum_{i} \delta_{i} \rho_{i} \neq 0\right.$ by Lemma (3)) and $P P$ exists and is unique.

Proposition 1: For any acceptable fixed transmission rate vector $R$ (respectively $\mathcal{C}$ ), there corresponds a unique minimum (component wise) power $P^{\prime \min }$ that satisfies the system (5) (respectively (9)). Moreover $P^{\prime \text { min }}=P P$.

Proof: As the problems (5) and (9) are equivalent, we only prove the proposition in the first case.

We extend the proof of Lemma 1 in [26] which only considers the single cell case. Suppose that there exists a feasible power assignment $P_{0}^{\prime}$ satisfying the constraints (5). We construct a sequence of power assignments $P_{i}^{\prime}$ where

$$
\left(p_{m, b}^{\prime}\right)_{i+1}=\delta_{m} r(m)\left(N(m, b)+C \sum_{m^{\prime} \neq m}\left(p_{m^{\prime}, b}^{\prime}\right)_{i}\right) .
$$

We have

$$
0 \leq\left(p_{m, b}^{\prime}\right)_{i+1} \leq\left(p_{m, b}^{\prime}\right)_{i} .
$$

Therefore the decreasing sequence converges to an assignment $P^{\prime \text { min }}$ satisfying $\forall n, P^{\prime \text { min }} \leq P_{n}^{\prime}$ component wise and

$$
\forall m, p_{m, b}^{\prime}{ }^{\min }=\delta_{m} r(m)\left(N(m, b)+C \sum_{m^{\prime} \neq m} p_{m^{\prime}, b}^{\prime \min }\right) .
$$

Lemma 5: Let $\mathcal{C}$ be a fixed acceptable transmission vector. The set of feasible power assignments satisfies:

$$
\left\{\begin{array}{rr}
0 \leq p_{s, t o t} \leq \overline{p_{s, t o t}}, & 1 \leq s \leq S, \\
\delta_{m} \rho(m)\left[C \sum_{m^{\prime}} \delta_{m^{\prime}} \rho\left(m^{\prime}\right)\left[N\left(m^{\prime}, b\right)-N(m, b)\right]\right. & \\
+N(m, b)] \leq\left(1-C \sum_{m^{\prime}} \delta_{m^{\prime}} \rho\left(m^{\prime}\right)\right) p_{m, b}^{\prime}, & 1 \leq m \leq M .
\end{array}\right.
$$

Proof: We consider the last inequality of system (9)

$$
p_{m, b}^{\prime} \geq \delta_{m} \rho(m)\left(N(m, b)+C \sum_{m^{\prime}} p_{m^{\prime}, b}^{\prime}\right),
$$

We can now consider cell $c$ separately, and reduce the unknown variables to the power and transmission rate within that cell only. We then sum over all mobiles of cell $c$ :

$$
\begin{aligned}
\left(\sum_{m^{\prime} \in c_{m}} p_{m^{\prime}, b}^{\prime}\right)\left(1-C \sum_{m^{\prime} \in c_{m}} \delta_{m^{\prime}} \rho\left(m^{\prime}\right)\right) \geq \\
\sum_{m^{\prime}} \delta_{m^{\prime}} \rho\left(m^{\prime}\right) N\left(m^{\prime}, b\right) .
\end{aligned}
$$


We finally combine this with (13) to obtain the second inequality of (12).

From Proposition 1 and Lemma 5, we get:

Lemma 6: For a given acceptable $R$ (respectively $\mathcal{C}$ ), $P^{\prime \text { min }}$ is given by $\forall m=1 \ldots M, p_{m, b}^{\prime}{ }^{\min }=\delta_{m} \rho(m) \times$

$$
\frac{N(m, b)+C \sum_{m^{\prime}} \delta_{m^{\prime}} \rho\left(m^{\prime}\right)\left[N\left(m^{\prime}, b\right)-N(m, b)\right]}{1-C \sum_{m^{\prime}} \delta_{m^{\prime}} \rho\left(m^{\prime}\right)}
$$

We finally conclude that:

Theorem 1: A rate vector $\mathcal{C}$ is acceptable if and only if it satisfies the two following conditions.

(C1) $\frac{M R_{m}}{1+\delta_{m} M R_{m}} \leq \rho(m) \leq \frac{P R_{m}}{1+\delta_{m} P R_{m}}$,

(C2) $\forall m, m=1 \ldots M, 0 \leq p_{s, t o t}^{m i n} \leq \overline{p_{s, t o t}}$, with $p_{s, t o t}^{\text {min }}$ defined by (2) and (15).

Proof: If $\mathcal{C}$ is feasible, then $(\mathrm{C} 1)$ is verified. Proposition 1 states that $P^{\prime \text { min }}$ is a solution of the system, so that $(\mathrm{C} 2)$ is also satisfied.

Equivalently, if condition (C2) is satisfied, then $P^{\prime \text { min }}$ is a solution vector (it satisfies the first and the third inequalities of (9)). Finally, (C1) is the last inequality of (9).

In this section, we focused on the continuous model and provided an equivalent system of equations based on a change of variables (Lemma 1). We then expressed a sufficient condition for the objective function to be concave (Lemma 2 and Remark 1). We showed three properties of this system (Proposition 1, Lemma 5 and Lemma 6). In particular, we showed that if a rate vector is acceptable (that is to say if it corresponds to at least one feasible power assignment), then all the corresponding power vectors are greater (component wise) than the feasible power vector $P^{\prime \text { min }}$ given by: $p_{m, b}^{\prime}{ }^{\min }=$ $\delta_{m} \rho(m) \times$

$$
\frac{N(m, b)+C \sum_{m^{\prime}} \delta_{m^{\prime}} \rho\left(m^{\prime}\right)\left[N\left(m^{\prime}, b\right)-N(m, b)\right]}{1-C \sum_{m^{\prime}} \delta m^{\prime} \rho\left(m^{\prime}\right)} .
$$

We finally concluded with a sufficient and necessary condition for a rate vector to be acceptable (Theorem 1).

\section{THE UPLINK CASE}

In this section, we apply the results of the previous section to the uplink case.

\section{A. Continuous case}

Let us consider a mobile $m$ in cell $c$. Let $g_{m, b}$ be the link gain between source $m$ (the mobile) and a destination $b$ (the base station). We assume that time intervals are sufficiently short for $g_{m, b}$ to be constant within the interval. $p_{m, b}$ is the power of the signal emitted by mobile $m$ to its corresponding base station. As mobile $m$ emits only one signal, we have: $p_{s, t o t}=p_{m, b}$. Finally, $\nu_{b}$ represents the thermal noise at destination. Then, the $S I R_{m}$ of mobile $m$ can be written:

$$
S I R_{m}=\frac{g_{b, m} p_{m, b}}{\nu_{b}+\sum_{m^{\prime} \text { in any cell }, m^{\prime} \neq m} g_{m^{\prime}, b} p_{m^{\prime}}} .
$$

We make the following approximating assumption that is frequently used for the uplink case (see e.g. [27]):

Hypothesis 1: The interference caused by mobiles from other cells is proportional to the interference due to the mobiles in cell $c$, i.e. there is a constant $\lambda$ such that $I_{\text {other }}=\lambda I_{\text {own }}$. In other words:

$$
\sum_{m^{\prime} \text { in any other cell }} g_{m^{\prime}, b} p_{m^{\prime}}=\lambda \sum_{m^{\prime} \text { in cell } c} g_{m^{\prime}, b} p_{m^{\prime}} .
$$

Under hypothesis 1 , the uplink can therefore be modeled with system (5) (equivalently with system (9)) with:

$$
C=\lambda+1, \quad N(m, b)=\nu_{b}, \quad p_{m, b}^{\prime}=g_{m, b} p_{m, b} .
$$

We then can apply the results of Section IV. In particular, we obtain (see also [16], [27]): $\forall m \in[1, M]$ :

$$
p_{m, b}^{\min }(\rho)=\frac{1}{g_{b, m}}\left(\frac{\nu_{b} \delta_{m} \rho(m)}{1-(1+\lambda) \sum_{m^{\prime}=1}^{N_{c}} \delta_{m^{\prime}} \rho\left(m^{\prime}\right)}\right) .
$$

Moreover, conditions (C2) of Theorem 1 is now:

$$
0 \leq \nu_{b} \delta_{m} \rho(m) \leq g_{b, m} \overline{p_{m, b}}\left(1-(1+\lambda) \sum_{m^{\prime} \in c_{m}} \delta_{m^{\prime}} \rho\left(m^{\prime}\right)\right) \text {. }
$$

Hence, the problem can then be summarized by: Find $\mathcal{C}$ that maximizes

$$
\begin{aligned}
& Z(\mathcal{C}):=\sum_{m=1}^{M} \frac{1}{1-\alpha}\left(\frac{\rho(m)}{1-\delta_{m} \rho\left(m^{\prime}\right)}\right)^{1-\alpha} \text { s.t. } \\
& \left\{\begin{array}{l}
\frac{M R_{m}}{1+\delta_{m} M R_{m}} \leq \rho(m) \leq \frac{P R_{m}}{1+\delta_{m} P R_{m}}, \\
0 \leq \nu_{b} \delta_{m} \rho(m) \leq g_{b, m} \overline{p_{m, b}}\left(1-(1+\lambda) \sum_{m^{\prime} \in c_{m}} \delta_{m^{\prime}} \rho\left(m^{\prime}\right)\right) .
\end{array}\right.
\end{aligned}
$$

Remark 2: The set of constraints is now a (convex) polytope.

Remark 3: A sufficient condition for all rate vectors to satisfy Lemma 3 is :

$$
\forall c, \quad(1+\lambda) \sum_{m^{\prime} \in c} \frac{\delta_{m^{\prime}} P R_{m^{\prime}}}{1+\delta_{m^{\prime}} P R_{m^{\prime}}}<1 .
$$

We conclude that for $\alpha \geq 2 w$ (and in particular for $\alpha \geq 2$, see Lemma 2 and Remark 1), the multicell problem of controlling jointly the power and the transmission rate can be reduced to a standard minimization problem with linear constraints and concave objective function that can be easily solved by either decentralized Lagrangian algorithms or efficient centralized methods based on SDP (Semi Definite Programming), see e.g. [28].

We finally note that for the single cell case, the above solution is an exact one.

\section{B. Further approximations for the uplink solution}

The condition $\alpha \geq 2 w$ does not cover the interesting case of $\alpha=0$ which corresponds to the problem of maximizing the global throughput. We therefore propose below two approximations, both applicable for all $\alpha \geq 0$. 
1) First approximation scheme: approximating the objective function.

One can approximate the objective function $Z(\rho)$ by $\sum_{m=1}^{M} \frac{(\rho(m))^{1-\alpha}}{1-\alpha}$, i.e. neglect the term $\delta_{m} \rho(m)$ in the denominator, as it is quite often much smaller than 1 (as mentioned before). With this new objective function replacing the previous one, we obtain a convex optimization problem for any $\alpha>0$ (except $\alpha=1$ ). We note that the constraints (and thus the set of feasible solutions) for this approximating method are the same as in the initial problem. We also note that the value obtained from this approximation is a lower bound for the original optimization problem.

2) Second approximation scheme: approximating the constrained set.

An alternative approximation can be obtained by considering the original formulation (5) in terms of the rate vector $R$ rather than $\mathcal{C}$, in which the objective function is already concave but the constraint set is not convex (see more details on this set in [22] that considers the single cell case). Our approximation then consists in replacing the last constraint in (5) by:

$$
\delta_{m} r(m) \leq \frac{g_{b, m} p_{m, b}}{\nu_{b}+\sum_{m^{\prime} \in c_{m}} g_{b, m^{\prime}} p_{m^{\prime}}}, \quad m=1, \ldots, M .
$$

We can now proceed as in Prop. 1 and consider the equality constraint instead of the inequality, which provides the minimal solution of (20), given by

$$
p_{m, b}^{\min }(r)=\frac{1}{g_{b, m}}\left(\frac{\nu_{b} \delta_{m} r(m)}{1-(1+\lambda) \sum_{m^{\prime} \in c_{m}} \delta_{m^{\prime}} r\left(m^{\prime}\right)}\right)
$$

(see the derivation of Eq. (18).

Substituting this into our new approximation problem, we obtain the equivalent optimization problem of maximizing $\sum_{m} \frac{r(m)^{1-\alpha}}{1-\alpha}$ over the set $\Pi_{\mathrm{app}}$ of vector $R$ satisfying

$\Pi_{\text {app }}\left\{\begin{array}{l}M R_{m} \leq r(m) \leq P R_{m}, \\ 0 \leq \nu_{b} \delta_{m} r(m) \\ \quad \leq g_{m, b} \overline{p_{m, b}}\left(1-(1+\lambda) \sum_{m^{\prime} i n c_{m}} \delta_{m^{\prime}} r\left(m^{\prime}\right)\right) .\end{array}\right.$

We see that the set of constraints is now a (convex) polytope.

Furthermore, let us consider a couple $\left(p^{\min }, R\right)$, where $p^{\text {min }}$ is computed in (21) for that $R$. If it is finite then the couple satisfies the third constraint in original constraint set $\Pi^{c}$. Therefore we replaced the set of constraints by a strict subset of that set. We conclude that the approximating problem gives in fact a lower bound on the throughput assignment for each $m$ and a lower bound for the objective function.

\section{The discrete model}

We finally briefly comment on the discrete model. The solution of the model (8) can be found in the same way as in [1], using a distributed algorithm based on Lagrangian relaxation. Alternatively, one can use the formulation (6). Its solution can follow a similar path as we had for the continuous case: first express for given transmission rates the corresponding minimum power that satisfies the constraint (4). Again the approximation (17) can be used to obtain explicit expressions for optimal power assignments for given transmission rates. This reduces to the same optimization problems we had before, with the same linear constraints, along with the new extra integrity constraint (7).

\section{DOWNLINK SOLUTION}

Following [29], we write a more precise expression for the signal to interference ratio (Equation (3)) that mobile $m$ connected to base station $b$ experiences: $S I R_{m, b}=$ $\frac{P_{b, m} h_{b, m}}{\nu_{m}+I_{\text {inter }}+I_{\text {intra }}}$, with $I_{\text {inter }}$ and $I_{\text {intra }}$ denoting respectively the intercell and the intracell interference at mobile $m$. We have :

$$
\left\{\begin{array}{l}
I_{\text {intra }}=\beta\left(P_{t o t, b}-P_{b, m}\right) h_{b, m}+(1-\beta) P_{S C H, b} h_{b, m}, \\
I_{\text {inter }}=\sum_{b^{\prime}=1, b^{\prime} \neq b}^{B} P_{t o t, b^{\prime}} h_{b^{\prime}, m} .
\end{array}\right.
$$

Equivalently $S I R_{m, b}=P_{b, m} /$

$\beta \sum_{m^{\prime} \neq m} p_{b, m^{\prime}}+P_{s c h}+\beta P_{c c h}+\frac{1}{h_{b, m}}\left[\nu_{m}+\sum_{b^{\prime}=1, b^{\prime} \neq b}^{B} P_{t o t, b^{\prime}} h_{b^{\prime}, m}\right]$.

where we denote by:

- $P_{b, m}$ the transmission power of base station $b$ to the Dedicated Physical Channel (DPCH) of mobile $m$,

- $P_{S C H, b}$ the power of the (non orthogonal) synchronization channel from base station $b$,

- $P_{C C H, b}$ the power of the (orthogonal) common channel from base station $b$,

- $P_{t o t, b}$ the total output power from base station $b$, given by

$$
P_{t o t, b}=\sum_{m^{\prime}=1}^{N_{c}} P_{b, m^{\prime}}+P_{C C H, b}+P_{S C H, b} .
$$

- $h_{b, m}$ the path gain from base station $b$ to mobile $m$,

- $\nu_{m}$ receiver's $m$ noise.

- $\beta$ the synchronization factor,

- $B$ the number of base stations.

Let us denote $F_{b, m}$ the ratio between the received intercell and intracell power, defined as

$$
F_{b, m}=\frac{I_{\text {intra }}}{I_{\text {inter }}} .
$$

Then $S I R_{m, b}=$

$$
\frac{P_{b, m}}{\left(1+F_{b, m}\right)\left(\beta \sum_{m^{\prime} \neq m} p_{b, m^{\prime}}+P_{s c h, b}+\beta P_{c c h, b}\right)+\frac{\nu_{m}}{h_{b, m}}} .
$$

As in [29], we shall further approximate $F_{b, m}$ by its average value $F$, and assume that $P_{C C H, b}$ and $P_{S C H, b}$ are the same for all base stations ( $b$ is then omitted). They are known 
parameters and are not subject to power control. Also, $\nu_{m}$ does not depend on $m$.

Then, the downlink joint transmission rates are now determined as the solution of problem (5) (or equivalently problem (9)) with:

$$
\begin{gathered}
N_{D}(b, m)=(1+F)\left(P_{s c h}+\beta P_{c c h}\right)+\frac{\nu}{h_{b, m}}, \\
C_{D}=(1+F) \beta \quad \text { and } \quad p_{m, b}^{\prime}=p_{b, m} .
\end{gathered}
$$

We thus obtain this optimization problem: Find $\mathcal{C}$ such that

$$
\left\{\begin{array}{l}
\frac{M R_{m}}{1+\delta_{m} M R_{m}} \leq \rho(m) \leq \frac{P R_{m}}{1+\delta_{m} P R_{m}}, \\
0 \leq p_{S C H}+p_{C C H}+\sum_{m \text { in cell } c} \delta_{m} \rho(m), \\
\frac{p_{b}^{\text {tot }} \geq \quad}{N_{D}(m, b)+C_{D} \sum_{m^{\prime}} \delta_{m^{\prime}} \rho\left(m^{\prime}\right)\left[N_{D}\left(m^{\prime}, b\right)-N_{D}(m, b)\right]} \\
\frac{1-C_{D} \sum_{m^{\prime}} \delta_{m^{\prime}} \rho\left(m^{\prime}\right)}{.} .
\end{array}\right.
$$

We can notice that for any values of $\delta, \rho, N$ and $C_{D}$ we obviously have:

$$
\begin{aligned}
& \sum_{m} \delta_{m} \rho(m)\left(N_{D}(m, b)+C_{D} \sum_{m^{\prime}} \delta_{m^{\prime}} \rho\left(m^{\prime}\right)\right. \\
& \left.\left[N_{D}\left(m^{\prime}, b\right)-N_{D}(m, b)\right]\right)=\sum_{m} \delta_{m} \rho(m) N_{D}(m, b)
\end{aligned}
$$

Moreover $1-C_{D} \sum_{m^{\prime}} \delta_{m^{\prime}} \rho\left(m^{\prime}\right)>0$ (Lemma (3)). Then: $-\left(p_{S C H}+p_{C C H}\right)\left(1-C_{D} \sum_{m^{\prime}} \delta_{m^{\prime}} \rho\left(m^{\prime}\right)\right) \leq 0 \leq$ $\sum_{m} \delta_{m} \rho(m) N_{D}(m, b)$

The optimization problem is finally: Find $\mathcal{C}$ that maximizes $Z(\mathrm{C}):=\sum_{m=1}^{M} \frac{1}{1-\alpha}\left(\frac{\rho(m)}{1-\delta_{m} \rho\left(m^{\prime}\right)}\right)^{1-\alpha}$ s.t.

$\left\{\begin{array}{l}\frac{M R_{m}}{1+\delta_{m} M R_{m}} \leq \rho(m) \leq \frac{P R_{m}}{1+\delta_{m} P R_{m}}, \\ \sum_{m \text { in cell } c} \delta_{m} \rho(m) N_{D}(m, b) \leq \overline{p_{b}^{t o t}}\left(1-C_{D} \sum_{m^{\prime}} \delta_{m^{\prime}} \rho\left(m^{\prime}\right)\right) .\end{array}\right.$

Then, once again we obtain a minimization problem with linear constraints. For $\alpha \geq 2 w$ the objective function is concave and therefore the general problem is convex and solvable in polynomial time.

\section{MACRO-DIVERSITY IN DOWNLINK}

Many UMTS systems use the possibility for a mobile to receive the signal from several stations. This is called macrodiversity. This prevents the signal from a base station from fading abruptly and as a consequence gives to the mobile a better quality of service.

For the power control part, we shall follow [29]. We consider below soft handover with two base stations or sectors $l$ and $s$; mobile $k$ has an active link to both stations. We assume maximum ratio combining where the sum of signal to interference ratio should add up to the target value $\delta_{k} r(k)$ :

$$
\delta_{k} r(k)=S I R_{k, l}+S I R_{k, s} .
$$

Assume that the link to station $s$ has better signal to interference ratio. Denote

$$
\Delta_{k}=\frac{S I R_{\text {worst link }}}{S I R_{\text {best link }}}=\frac{S I R_{k, l}}{S I R_{k, s}} \leq 1 .
$$

In order to solve the joint power and transmission-rate control, we proceed as in [29]. We make the simplifying assumption that $\Delta_{k}$ does not depend on $k$ (we can take the average value among mobiles that are in soft handover).

Let $I$ be the set of mobiles in cell $s$ that do not experience handover. For such mobiles, we have $\delta_{i} r(i)=S I R_{i, s}$ with $S I R_{i, s}$ given by Equation (24).

Let $j$ be a mobile in soft handover. Then, $\delta_{j} r(j)=(1+$

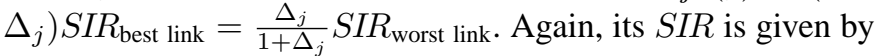
Equation (24). Then, we can distinguish two sets of mobiles : $J$ is the set of mobiles which best link is with base station $s$, and $K$ is the set of mobiles also experiencing soft handover, but which worst link is with base station $s$.

Equation (23) becomes:

$P_{t o t, b}=\sum_{i \in I} P_{i, b}+\sum_{j \in J} P_{j, b}+\sum_{k \in K} P_{k, b}+P_{C C H, b}+P_{S C H, b}$.

Define:

$$
\begin{cases}N_{I}(b, m)=N_{D}(b, m), & C_{I}=C_{D} \\ N_{J}(b, m)=\frac{1}{\Delta+1} N_{D}(b, m), & C_{J}=\frac{1}{\Delta+1} C_{D} \\ N_{K}(b, m)=\frac{\Delta}{\Delta+1} N_{D}(b, m), & C_{K}=\frac{\Delta}{\Delta+1} C_{D}\end{cases}
$$

Note, in contrast that the authors of [29] do not distinguish between the sets $J$ and $K$, that is why their equation differs. Also, they assume that the number of mobile in all cell is constant, and that $\forall i, j, \delta_{i} r(i)=\delta_{j} r(j)$.

Let $i(m)$ be the set that $m$ is belonging to $(i(m) \in$ $\{I, J, K\})$. We get the following optimization problem for determining the transmission rates:

Find $\mathcal{C}$ that $\sum_{m=1}^{M} \frac{1}{1-\alpha}\left(\frac{\rho(m)}{1-\delta_{m} \rho\left(m^{\prime}\right)}\right)^{1-\alpha}$ s.t.

$$
\left\{\begin{array}{l}
\frac{M R_{m}}{1+\delta_{m} M R_{m}} \leq \rho(m) \leq \frac{P R_{m}}{1+\delta_{m} P R_{m}} \\
\sum_{m \text { in cell } c} \delta_{m} \rho(m) N_{i(m)}(b, m) \\
\leq \overline{p_{b}^{t o t}}\left(1-C_{i(m)} \sum_{m^{\prime}} \delta_{m^{\prime}} \rho\left(m^{\prime}\right)\right) .
\end{array}\right.
$$

We thus obtain again an optimization problem with concave objective function for all $\alpha \geq 0, \alpha \neq 1$ and linear constraints, which is standard to solve and has efficient solutions. 


\section{NUMERICAL TESTS}

In the following, we show some of the results obtained using the program (19) for the uplink. For $M$ mobiles, we consider a single cell and set $\forall m, \delta_{m}=1, \nu=1, \overline{p_{m, b}}=1 / M$, $M R_{m}=1 / 4 M$, and $P R_{m}=1$. The position of the mobiles is taken at random in the $[-1 ;+1] \times[-1 ;+1]$ square, and the gain $g_{b, m}$ is equal to $1 / d_{m}^{2}$, where $d_{m}$ is the distance of the mobile to the center of the square. We take $M=50$.

On figures 1 and 2 we show some results obtained for $\alpha$ equal to 0 and 1 corresponding to the global optimization and the proportional fairness respectively. The base station is represented as a black circle in the middle of the figure, and the mobiles are represented with circles centered at their location, and whose radius is proportional to the throughput assigned. Clearly we see that the mobiles closer to the base station tend to receive more bandwidth. Also many subtle differences appear between the two fairness criteria. In particular, proportional fairness allocate no mobile to its maximum throughput demand $P R$ and redistributes the bandwidth to in-between users. Further users still receive the minimum bandwidth $M R$.

\section{CONCLUding REMARKS AND EXTENSIONS}

In this paper we addressed the problem of joint transmission rate and power control in wireless networks so as to be both fair and optimal.

A question not addressed here is how to achieve these throughputs in practice if packet mode is used, or in other words, how to schedule packets in order to achieve the throughputs that were fairly assigned. This question has been well studied see e.g. [7]-[9].

The paper is in line with many references [4], [6], [30], [31] that considered the throughput as the object to be fairly assigned, in other networking contexts. In the fairness analysis, one may consider the case in which the utility corresponding to the transmission rates should be fairly assigned, rather than directly the throughputs. Indeed, since utility represents the degree of satisfaction as a function of the assigned throughput, which may be application dependent, assigning the same throughput to two applications might be highly unfair. In fact, mathematical frameworks for defining fairness indeed exist, within the area of cooperative game theory, and they always relate to utilities. The central concept of this type that has been applied to fair resource allocation problems is the so called Nash Bargaining solution [28], [31], [32]; it turns out to agree with the proportional fairness concept when utilities are linear. If $f_{j}(r(j))$ is the utility for mobile $j$ to have a transmission rate of $r(j)$, then the Nash Bargaining Solution is given by the solution of the optimization problem:

$$
\left(P_{J}\right) \quad \max \prod_{i=1}^{M}\left(f_{i}(r(i))-f_{i}\left(M R_{i}\right)\right),
$$

where the maximization is over the appropriate constrained set $\left(\Pi^{c}\right.$ in the continuous model, $\Pi^{a p p}$ for the corresponding approximating problem, and $\Pi^{d}$ in the discrete problem), see [28]. For real-time voice applications, $f_{i}$ are typically concave functions over the interval $\left[M R_{i}, P R_{i}\right]$, which implies that the objective function in the above problem is concave. In particular, if we consider use the approximating approach to compute bounds for the continuous model and solve $P_{j}$ over the constrained set $\Pi^{a p p}$, this is then again a standard concave optimization problem with linear constraint for which many efficient (polynomial) methods exist. In particular, several solution methods are proposed in [28] for such problems if we express the utilities using quadratic functions.

\section{ACKNOWLEDGMENT}

We wish to thank Mr. Jean-Marc Kelif and Dr. Zwi Altman for their many useful discussions.

\section{REFERENCES}

[1] Z. R. S. L. Kim and J. Zander, "Combined power control and transmission selection in cellular networks," in Proc. of IEEE Vehicular Technology Conference, Fall 1999.

[2] "Traffic management specification," The ATM forum Technical Committee, April 1996, version 4.0.

[3] D. Bertsekas and R. Gallager, Data Networks. Prentice-Hall, 1987.

[4] A. M. F. P. Kelly and D. Tan, "Rate control for communication networks: shadow prices, proportional fairness and stability," Journal of the Operational Research Society, vol. 49, 1998.

[5] J. Crowcroft and P. Oechslin, "Differentiated end-to-end internet services using a weighted proportional fair sharing tcp," Computer Communications Review, vol. 28, no. 3, pp. 53-67, July 1998.

[6] J. Mo and J. Walrand, "Fair end-to-end window-based congestion control," in Proc. of SPIE International Symposium on Voice, Video and Data Communications, 1998.

[7] S. L. V. Bharghavan and T. Nandagopal, "Fair queuing in wireless networks, issues and applications," IEEE Personal Communications, vol. 6, no. 1, Feb 1999.

[8] G. Miklós and S. Molnár, "Fair allocation of elastic traffic for a wireless base station," in Proc. of IEEE Globecom, Rio de Janeiro, Dec. 1999, pp. 1673-1678.

[9] V. B. S. Lu and R. Srikant, "Fair scheduling in wireless packet networks," IEEE/ACM Trans. on Networking, vol. 7, no. 4, pp. 473489, 1999.

[10] C. T. E. Altman, J. Galtier, "Fair power and transmission rate control in wireless networks," in IEEE Globecom, Taipei, Taiwan, Nov. 2002.

[11] S. Oh and K. M. Wasserman, "Optimality of greedy power control and variable spreading gain in multiclass cdma mobile networks," in Proc. of Mobicom, Seattle, Washington, USA, 1999, pp. 102-112.

[12] - "Dynamic spreading gain control in multiservice cdma networks," IEEE J. Selected Area in Comm., vol. 17, no. 5, pp. 918-927, May 1999.

[13] _ "Adaptive resource allocation in power constrained cdma mobile networks," in Proc. IEEE WCNC, Sept. 1999, pp. 510-514.

[14] W. S. W. C. W. Sung, "Power controll and rate management for wireless multimedia cdma systems," IEEE Trans. on Communications, vol. 49, no. 7, pp. 1215-1226, Jul 2001.

[15] S. Ramakrishna and J. M. Holtzman, "A scheme for throughput maximization in a dual-class cdma system," IEEE Journal Selected Areas in Comm., vol. 16, pp. 830-844, Aug. 1998.

[16] E. Altman, "Capacity of multi-service cdma cellular networks with besteffort applications," INRIA," Research Report, March 2002.

[17] J. W. M. D. Zhao, X. Shen, "Quality-of-service support by power and rate allocation in mc-cdma systems," in Proc. IEEE GLOBECOM, Nov. 2001, pp. 604-608.

[18] J. W. M. M. Cheung, "Resource allocation in wireless networks based on joint packet/call levels qos constraints," in Proc. of IEEE Globecom, Nov 2000, pp. 271-275.

[19] S. Z. J. W. Mark, "Power control and rate allocation in multirate wideband cdma systems," in Proc. IEEE WCNC, Sept 2000, pp. 168172.

[20] J. W. M. L. Xu, X. Shen, "Performance analysis of adaptive rate and power control for data service in ds-cdma systems," in Proc. of IEEE GLOBECOM, Nov. 2001, pp. 627-631.

[21] R. Berry, "Power and delay trade-offs in fading channels," Ph.D. dissertation, Massachusets Institute of Technology, June 2000. 


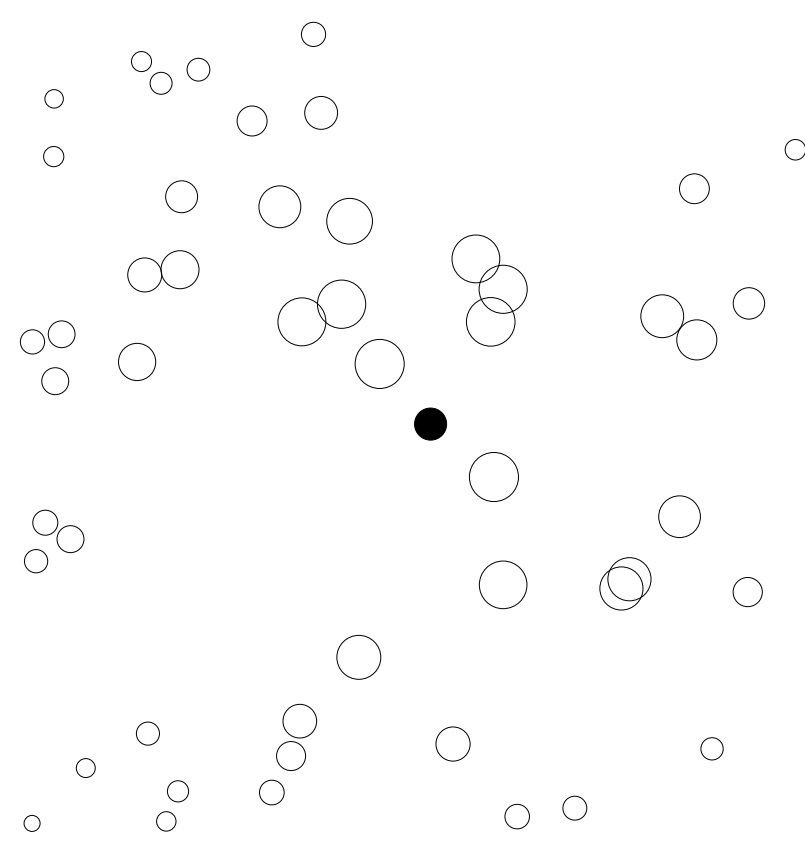

Fig. 1. Throughput distribution with $\alpha=0$.

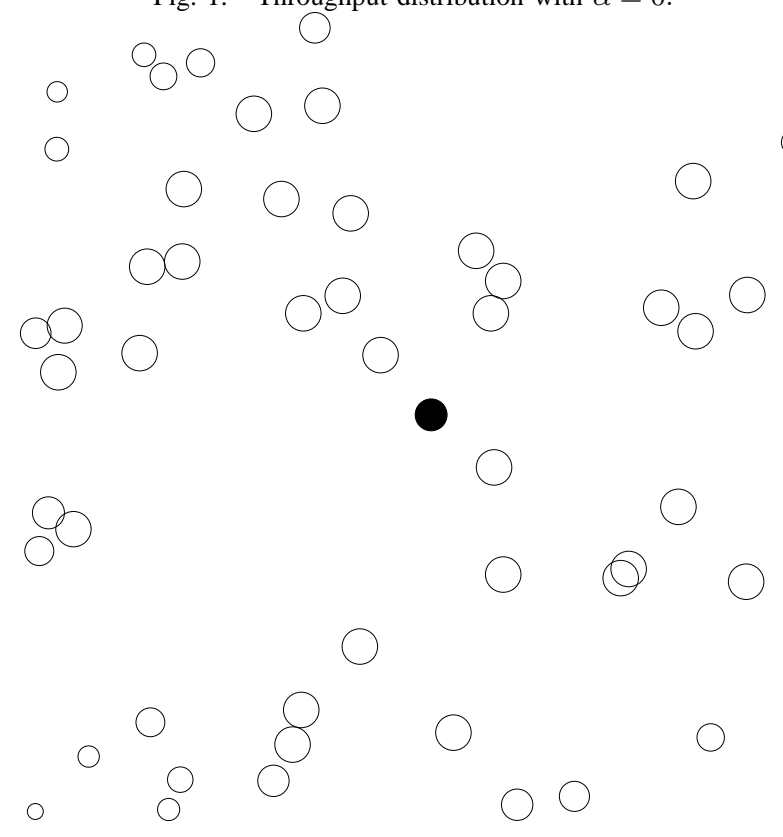

Fig. 2. Throughput distribution with $\alpha=1$.

[22] R. Leelahakriengkrai and R. Agrawal, "Scheduling in multimedia dscdma wireless networks," Technical Report ECE-99-3, July 1999, university of Wisconsin - Madison.

[23] F. C. A. Baiocchi and C. Martello, "Optimizing the radio resource utilization of multiaccess systems with a traffic-transmission quality adaptive packet scheduling," Computer Networks, vol. 38, pp. 225-246, 2002.

[24] R. V. M. Ferracioli, V. Tralli, "Channel adaptive scheduling for a wideband tdd/tcdma wireless system under heterogeneous traffic conditions," Computer Networks, vol. 38, pp. 207-223, 2002.

[25] H. Holma and A. Toskala, WCDMA for UMTS. J. Wiley \& Sons, 2001, revised Edition.

[26] D. Ayyagari and A. Ephremides, "Power control for link quality protection in cellular ds-cdma networks with integrated (packet and circuit) services," in Proc. of Mobicom'99, Seattle Washington, USA, 1999, pp.
96-102.

[27] J. Laiho and A. Wacker, "Radio network planning process and methods for wcdma," Ann. Telecommun., vol. 56, no. 5-6, 2001.

[28] C. Touati, E. Altman, and J. Galtier, "On fairness in bandwidth allocation," INRIA Research Report, Sept. 2001.

[29] K. Hiltunen and R. D. Bernardi, "Wcdma downlink capacity estimation," in Proc. of VTC'2000, 2000, pp. 992-996.

[30] L. Massouillé and J. W. Roberts, "Bandwidth sharing and admission control for elastic traffic," Telecommunication Systems, 2000.

[31] R. R. M. H. Yaiche and C. Rosenberg, "A game theoretic framework for bandwidth allocation and pricing in broadband networks," IEEE/ACM Transactions on Networking, vol. 8, no. 5, pp. 667-677, 2000.

[32] R. Mazumdar and C. Douligeris, "Fairness in network optimal flow control: Optimality of product forms," IEEE Trans. on Comm., vol. 39, pp. 775-782, May 1991. 